\title{
O diabetes hiperlábil existe como entidade clínica?
}

\author{
Does brittle diabetes exist as a clinical entity?
}

Freddy Goldberg Eliaschewitz', Denise Reis Franco²

\begin{abstract}
RESUMO
A melhor compreensão das causas da instabilidade dos níveis da glicemia em pacientes com diabetes melito tipo 1 (DMT1) e a disponibilidade de novas alternativas para enfrentá-la com sucesso, como a bomba de infusão contínua de insulina e os análogos das insulinas, tornaram relevante o questionamento sobre a existência do diabetes hiperlábil como uma entidade bem como a necessidade de defini-lo. O presente artigo pretendeu descrever o conceito de diabetes hiperlábil à luz dos novos avanços na terapia do DMT1 e propor critérios objetivos para a quantificação da labilidade da glicemia. Arq Bras Endocrinol Metab. 2009;53(4):466-9.
\end{abstract}

'Núcleo de Terapia Celular e Molecular (Nucel), Universidade de São Paulo (USP); Centro de Pesquisas Clínicas (CPClin); Hospital Heliópolis; Centro de Pesquisa Médica NotreDame Intermédica Sistema de Saúde, São Paulo, SP, Brasil ${ }^{2}$ CPClin; Centro de Pesquisa Médica Notre-Dame Intermédica Sistema de Saúde, São Paulo, SP, Brasil

Correspondência para: Freddy Goldberg Eliaschewitz Rua Goiás, 91 01244-030 - São Paulo, SP, Brasil freddy.g@uol.com.br

Recebido em 14/Jul/2008 Aceito em 21/Jan/2009
Descritores

Diabetes hiperlábil; hipoglicemias inadvertidas; hipoglicemias não percebidas; transplante de ilhotas; terapia tissular; diabetes melito tipo 1

\section{ABSTRACT}

The best comprehension about the instability of the glycemia levels in type 1 diabetes mellitus (T1DM) patients and the availability of new alternatives to successfully control it, like insulin pump therapy and the insulin analogues, underlined the questions about the brittle diabetes existence as a clinical entity as well as the necessity of define it. The aim of this article was to describe the concept of brittle diabetes in the light of the latest advances in the treatment of type 1 diabetes and propose objective criteria to evaluate the level of glucose liability. Arq Bras Endocrinol Metab. 2009;53(4):466-9.

Keywords

Brittle diabetes; hypoglycemia unawareness; islet transplantation; tissue therapy; type 1 diabetes

\section{INTRODUÇÃO}

$\mathrm{O}$ $s$ recentes avanços da monitoração da glicemia permitiram a identificação de pacientes com diabetes melito tipo l (DMTl), que, apesar das novas modalidades de insulinoterapia, apresentam labilidade no controle glicêmico (1). O DMTl com esse comportamento clínico foi recentemente designado diabetes hiperlábel ou instável.

A expressão "brittle diabetes" (diabetes hiperlábil, lábil ou instável) foi cunhada em 1934 por R. T. Woodyatt, que nunca publicou uma definição precisa desse termo, mas viu seu uso se disseminar, sendo imediatamente utilizado para identificar pacientes diabéticos cujas glicemias oscilavam exageradamente, de modo imprevisível e sem explicação aparente (2).

Postulou-se, na época, que, nesses pacientes, a insulina teria uma farmacocinética alterada e que a hiper- labilidade seria, portanto, uma condição inteiramente explicada por razões físicas. Nos anos 1950, com o desenvolvimento da Medicina Psicossomática, essa população foi estudada, sendo encontrada nela uma alta incidência de distúrbios emocionais. Os diabéticos hiperlábeis foram, então, classificados em dois grupos: os de causa orgânica e os de causa psicológica $(2,3)$.

Tal visão perdurou até os anos 1970, quando o melhor conhecimento dos mecanismos que regulam a homeostase da glicose tornou evidente que as alterações emocionais podem ter, por si só, um grande impacto sobre o controle glicêmico, que ocorre principalmente por meio da ação de mediadores hormonais, como as catecolaminas e os glicocorticoides. Assim, verificou-se que essa classificação era algo arbitrária, já que fatores orgânicos e emocionais frequentemente interagiam como causa da instabilidade metabólica (4). 
Além disso, a elucidação do fenômeno de Somogyi evidenciou o papel das hipoglicemias recorrentes como causadoras de hiperglicemias de difícil controle (rebote). Esse fenômeno ocorre mesmo quando as hipoglicemias são assintomáticas. Os anos 1980 foram marcados pelo reconhecimento da neuropatia autonômica como causa da resposta deficiente na secreção dos hormônios contrarregulatórios durante a vigência de um episódio hipoglicêmico, explicando, assim, o mecanismo da ocorrência de episódios de hipoglicemia não percebida (5).

Já nos anos 1990, verificou-se que os próprios episódios hipoglicêmicos de repetição poderiam ser responsáveis pela insuficiência autonômica. Nessa época, assim, foi desvendado o mecanismo pelo qual as hipoglicemias de repetição criavam um círculo vicioso, pois, ao dessensibilizar a resposta adrenérgica, estas permitiam a ocorrência de novos episódios assintomáticos de hipoglicemia, que resultavam em eventos de rebote inesperados, causando a instabilidade metabólica. Mais recentemente, verificou-se que essa dessensibilização era frequentemente reversível se o paciente pudesse permanecer por seis semanas livre das hipoglicemias (6).

Com isso, ficava claro que a labilidade não é uma característica indelével que marca um grupo de indivíduos diabéticos. Pelo contrário, fases de instabilidade induzidas por um motivo qualquer, caso não sejam contidas a tempo, podem transformar um paciente antes estável em um com diabetes hiperlábil. Por outro lado, se um indivíduo com diabetes tido como hiperlábil puder ter seu controle mantido estável por tempo suficiente, poderá, a partir da recuperação de sua percepção das hipoglicemias, ter seu quadro de hiperlabilidade resolvido (6).

Em 1997, baseado no fato de que tanto esse mecanismo quanto o risco que o paciente corre dependem mais da intensidade da labilidade do que da etiologia, Tattersall propôs uma definição funcional de diabetes hiperlábil (2). Diabetes hiperlábil é aquele paciente que, pela frequência ou intensidade dos episódios de hipo ou hiperglicemia, se vê impedido de ter um ritmo de vida no qual possa desempenhar as atividades normais do cotidiano, independentemente da causa da hiperlabilidade.

No entanto, nem todos os autores concordam com essa definição. A melhor compreensão das causas da instabilidade dos níveis da glicemia e a disponibilidade de novas alternativas para enfrentá-la com sucesso, tais como a bomba de infusão contínua de insulina e os análogos das insulinas, têm levado ao questionamento sobre a real existência do diabetes hiperlábil como uma entidade e, portanto, sobre a necessidade de defini-la.
Scheiner (7), por exemplo, relata que "[...] embora rotular alguém com o diagnóstico de diabetes hiperlábil possa trazer certo alívio para o paciente, por oferecer uma explicação para a flutuação inesperada da glicemia, [...] pode na realidade fazer com que se deixe de investigar as causas reais da labilidade, que quase sempre podem ser encontradas, e este é o primeiro e mais importante passo rumo à correção do problema" (7).

Para além de qualquer discussão sobre a necessidade e a maneira de definir o diabetes hiperlábil, está o fato de que há um grupo de indivíduos com diabetes de controle muito difícil e que é marcado por um risco maior de complicações - tanto imediato, pelas hipoglicemias, quanto em longo prazo, pelas complicações crônicas - mesmo que não constitua uma entidade perfeitamente definida. Esta é a razão pela qual determinadas condutas terapêuticas, como transplantes, são reservadas a esse grupo de pacientes.

\section{PERFIL DO INDIVÍDUO COM DIABETES HIPERLÁBIL}

A hiperlabilidade se manifesta como hiperglicemia persistente $(70 \%)$, associada ou não a cetoacidose recorrente $(52 \%)$, ou ainda como hipoglicemia recorrente (12\%). Entre $12 \%$ e $24 \%$ dos pacientes têm formas mistas de instabilidade.

Quando comparados a um grupo-controle representado por indivíduos com diabetes estável, os indivíduos com diabetes hiperlábil são predominantemente do sexo feminino ( $86 \%$ versus $45 \%$ ), mais jovens, e usam, em média, o dobro da dose diária de insulina.

Os distúrbios psicossociais são diagnosticados em $72 \%$ dos pacientes hiperlábeis, o que ocorrem em apenas $17 \%$ dos pacientes estáveis. Fases de instabilidade são identificadas como induzidas pelos próprios pacientes em $40 \%$ dos casos no grupo dos hiperlábeis e em $2 \%$ no grupo-controle (8).

\section{CAUSAS DE HIPERLABILIDADE}

As causas de hiperlabilidade são variadas e podem estar ligadas ao modo ou tipo de tratamento, bem como a patologias e complicações associadas $(7,9)$.

\section{Causas educacionais e econômicas: uso incorreto da insulina NPH e regular}

O início da ação, o pico de efeito e a duração dessas insulinas dependem do local onde são injetadas. Elas são 
absorvidas mais rapidamente se aplicadas no abdômen e mais lentamente nas nádegas. Assim, para que o efeito seja mais reprodutível, o paciente deve aplicar a insulina do mesmo período sempre na mesma região. Por exemplo, o paciente deve aplicar a NPH da manhã sempre no abdômen e a da noite sempre nas nádegas, respeitando a distância mínima de um dedo da aplicação anterior.

Já os análogos da insulina são absorvidos no mesmo ritmo, independentemente do local da aplicação. A falta de rotação adequada dos locais de injeção é um fator comum de instabilidade. No entanto, talvez o erro mais comum seja a falta de homogeneização das insulinas em suspensão (NPH, por exemplo). A falta de agitação do frasco ou da caneta por cerca de dez vezes antes da aplicação da insulina leva a uma variação da dose real em torno de $45 \%$, entre uma aplicação e outra.

Além disso, o paciente deve saber ajustar as doses de acordo com a atividade física e com o tipo/quantidade da refeição, e aplicá-las no intervalo de tempo correto em relação a elas. Para que o paciente possa fazer a aplicação das doses corretas, é imprescindível que ele monitore as glicemias adequadamente, já que a dose a ser aplicada depende também dos níveis pré-prandiais da glicemia. A falta de monitorização adequada, algumas vezes por falta das tiras reagentes, pode não só resultar em um mau controle do diabetes, mas também levar o paciente à hiperlabilidade autoinflingida.

\section{Causas orgânicas}

Outras causas comuns de hiperlabilidade são as relacionadas às alterações hormonais da puberdade, menstruação e gestação, ou ainda as associadas a alterações do comportamento alimentar, como anorexia nervosa, e às síndromes disabsortivas ou a complicações do próprio diabetes, como gastroparesia e neuropatia autonômica. A apneia do sono e o uso de medicações capazes de induzir a resistência à insulina também são causas relativamente frequentes de hiperlabilidade (11).

\section{Causas menos frequentes}

Outras causas incluem a insuficiência adrenal primária ou secundária, a resistência à insulina mediada por anticorpos (imunológica) e as alterações farmacocinéticas da insulina injetada, por degradação proteolítica da insulina no subcutâneo.

Quando um indivíduo com diabetes hiperlábil não tem a remissão desse quadro, apesar da identificação e do tratamento da causa da hiperlabilidade, ou quando a causa permanece sem diagnóstico, o transplante de ilhotas pode ser considerado. Essa indicação pode ser justificada pelo maior risco que esses pacientes correm, principalmente quando episódios recorrentes de hipoglicemias não percebidas fazem parte do quadro clínico. Nessa condição, a mortalidade pode ser até 16 vezes maior do que a apresentada por diabéticos tipo 1 estáveis (12).

Como o risco está diretamente ligado ao grau de labilidade, métodos mais objetivos de quantificá-la são necessários.

\section{Quantificando a hiperlabilidade}

A hiperlabilidade compreende diferentes aspectos: a gravidade e a frequência dos episódios de hipoglicemia - principalmente os não percebidos, que são quantificados pelo escore HYPO, a velocidade de variação da glicemia quantificada pelo fator LI (labilidade) e a amplitude da variação da glicemia quantificada pelo MAGE.

Todos os indivíduos com DMTl têm certo grau de labilidade no seu controle glicêmico e apresentam episódios de hipo/hiperglicemia com alguma frequência. Desse modo, a maneira adequada de quantificar a labilidade entre grupos é tomar como referência a flutuação da glicemia que ocorre normalmente numa população de pacientes considerada estável, que julga não estar necessitada de terapia adicional e que avalia que o diabetes não os impede de desempenhar as atividades normais do cotidiano. Foi em um grupo selecionado dessa maneira que Ryan e cols. encontraram os cem pacientes do seu grupo-controle que consentiram em participar da pesquisa. Esses pacientes foram comparados a 51 pacientes que se inscreveram no programa de transplante de ilhotas da Universidade de Alberta (13). A quantificação da hiperlabilidade nesse estudo foi baseada no cálculo de três índices. O escore HYPO é calculado a partir do número de episódios de hipoglicemia em um acompanhamento de quatro semanas, com ponderações específicas para frequência, severidade e grau de percepção das hipoglicemias. A pontuação é maior quando o episódio é assintomático. Faz parte desse escore um dado fornecido retrospectivamente pelo paciente, que é o número de episódios hipoglicêmicos severos de que ele se recorda de ter tido no último ano. O outro fator, LI, que é obtido a partir das determinações da glicemia nas quatro semanas de registro, é dado pela fórmula: 


\section{N}

LI mmol//2 $x$ semana $^{-1}=\Sigma\left(\text { Gluc }_{n}-\text { Gluc }_{n+1}\right)^{2} /\left(h_{n+1}-h_{n}\right)$

$n=1$

onde:

$\mathrm{N}=$ total das medições da glicemia obtidas na semana;

Gluc $_{n}=$ é a enésima determinação da glicemia da semana obtida na hora $h_{n}$

Ambos os índices foram validados estatisticamente; além disso, após a obtenção dos índices, as determinações das glicemias de quatro semanas foram apresentadas a dois diabetologistas independentes, que desconheciam o estudo, para a classificação clínica do grau de labilidade em escores variando de 0 (perfeitamente estável) a 10 (extremamente lábil). Houve boa concordância entre o escore calculado e a avaliação clínica.

O terceiro parâmetro avaliado é o MAGE (amplitude média das excursões glicêmicas) baseado nas flutuações da glicemia em sete determinações diárias, por dois dias consecutivos.

O escore médio do HYPO no grupo-controle foi 143, comparado a 722 no grupo considerado lábil. O LI foi 223 no grupo-controle, comparado a 497 no grupo lábil. As diferenças foram estatisticamente significativas e foi possível correlacionar os índices a eventos que realmente refletem o aumento de risco, como o número de internações, comas e acidentes (13).

Portanto, é possível medir o grau de labilidade de modo mais objetivo e, assim, selecionar os pacientes destinados a terapias mais sofisticadas, como as bombas de infusão contínua ou invasivas, estratificando-os pelo risco.

\section{CONCLUSÕES}

A condição de diabetes hiperlábil não é uma marca indelével, que representa um subtipo específico de DMTl, mas uma condição clínica quantificável e frequentemente reversível, na medida em que suas causas são identificadas e abordadas com medidas terapêuticas apropriadas. A disponibilidade de novas formas de insulinoterapia tem o potencial de reduzir o número de pacientes (15) com indicação para terapia de reposição celular, a qual, pelo seu alto custo e pela necessidade de imunossupressão, deve ser reservada àqueles que realmente não têm outra alternativa de tratamento.

Declaração: os autores declaram não haver conflitos de interesse científico neste artigo.

\section{REFERÊNCIAS}

1. Maia FF, Araújo LR. A hipoglicemia silenciosa é parte do controle glicêmico ideal em pacientes com DM1? - Tempo de hipoglicemia pelo CGMS versus média glicêmica. Arq Bras Endocrinol Metabol. 2008;52(6):994-1000.

2. Tattersall RB. Brittle diabetes revisited: the Third Arnold Bloom Memorial Lecture. Diabet Med. 1997;14(2):99-110.

3. Brosig B, Leweke F, Milch W, Eckhard M, Reimer C. Psychosocial predictors of metabolic instability in brittle diabetes - A multivariate time series analysis. Psychother Psychosom Med Psychol. 2001;51(6):232-8.

4. Dutour A, Boiteau V, Dadoun F, Feissel A, Atlan C, Oliver C. Hormonal response to stress in brittle diabetes. Psychoneuroendocrinology. 1996;21(6):525-43.

5. Campbell IW. The Somogyi phenomenon. A short review. Acta Diabetol Lat. 1976;13(1-2):68-73.

6. Cryer PE. Diverse causes of hypoglycemia-associated autonomic failure in diabetes. N Engl J Med. 2004;350(22):2272-9.

7. Scheiner G. The myth of brittle diabetes. Diabetes Self Manag. 2003;20(4):64, 66, 69-70, 73 .

8. Tattersall RB. Brittle diabetes. Clin Endocrinol Metab. 1977;6(2):403-19.

9. Eiber R, Berlin I, Grimaldi A, Bisserbe JC. Insulin-dependent diabetes and psychiatric pathology: general clinical and epidemiologic review. Encephale. 1997;23(3):351-7.

10. Schade DS, Drumm DA, Duckworth WC, Eaton RP. The etiology of incapacitating, brittle diabetes. Diabetes Care 1985;8(1):12-20.

11. Schade DS, Burge MR. Brittle diabetes: etiology and treatment. Adv Endocrinol Metab. 1995;6:289-319.

12. Nielsen S, Emborg C, Molbak AG. Mortality in concurrent type 1 diabetes and anorexia nervosa. Diabetes Care. 2002;25(2):309-12.

13. Ryan EA, ShandroT, Green K, Paty BW, Senior PA, Bigam D, et al. Assessment of the severity of hypoglycemia and glycemic lability in type 1 diabetic subjects undergoing islet transplantation. Diabetes. 2004;53(4):955-62.

14. Selam JL. How to measure glycemic instability? Diabetes Metab. 2000.26(2):148-51.

15. Shalitin S, Phillip M. Hypoglycemia in type 1 diabetes: a still unresolved problem in the era of insulin analogs and pump therapy. Diabetes Care. 2008;31 Suppl 2:S121-4 